\title{
P. VALLOIS
}

\section{S. TAPIERO}

\section{Moments of an amplitude process in a random walk and approximations : computations and applications}

Revue française d'automatique, d'informatique et de recherche opérationnelle. Recherche opérationnelle, tome 29, n 1 (1995), p. 1-17.

<http://www.numdam.org/item?id=RO_1995_29_1_1_0>

(C) AFCET, 1995, tous droits réservés.

L'accès aux archives de la revue "Revue française d'automatique, d'informatique et de recherche opérationnelle. Recherche opérationnelle » implique l'accord avec les conditions générales d'utilisation (http://www.numdam.org/ legal.php). Toute utilisation commerciale ou impression systématique est constitutive d'une infraction pénale. Toute copie ou impression de ce fichier doit contenir la présente mention de copyright.

\section{Numdam}

Article numérisé dans le cadre du programme

Numérisation de documents anciens mathématiques

http://www.numdam.org/ 


\title{
MOMENTS OF AN AMPLITUDE PROCESS IN A RANDOM WALK AND APPROXIMATIONS: COMPUTATIONS AND APPLICATIONS (*)
}

\author{
by P. VAllois $\left({ }^{1}\right)$ and C. S. TAPIERO $\left({ }^{2}\right)$
}

\begin{abstract}
This paper evaluates the first two moments of the inverse of the range process in a symmetric Bernoulli random walk. Applications providing a strong motivation for this problem are considered.
\end{abstract}

Keywords: Stochastic processes, range process, quality control.

Résumé. - Cet article évalue les deux premiers moments de l'inverse du processus de l'amplitude d'une marche aléatoire symétrique. Des applications motivant ce problème sont considérées.

Mots clés : Processus stochastique, processus de l'amplitude, contrôle de qualité.

\section{INTRODUCTION}

The purpose of this paper is to consider the range process for a discrete random walk. This problem is of considerable usefulness in the control of variability. For example, it can be used to test the variability of a random walk, in providing statistical tests for the detection of outliers and in general in testing the variability of stochastic processes (such as stock prices, exchange rate mechanisms, control of production processes, etc.). Applications are considered in subsequent papers, although some simple examples are treated here. This paper derives both the probability generating function as well as its first two moments. A single parameter standardized version is obtained whose mean is 1 and whose variance converges quickly to $1 / 3$.

(*) Received January 1994.

( $\left.{ }^{1}\right)$ Département de Mathématiques, Université de Nancy-I, B.P. 239, 54506 Vandœuvre-lèsNancy Cedex, France.

(2) ESSEC, B.P. 105, 95021 Cergy Pontoise, France. 


\section{THE RANDOM WALK MODEL}

Consider a symmetric Bernoulli random walk and let $x_{i}, i=1,2, \cdots$ be the record of this process. Namely, at any time $t+1$, the process can either increase or decrease by a unit with the same probability, or

$$
x_{t+1}=x_{t}+\epsilon_{t}, \quad x_{0}=0
$$

with

$$
\epsilon_{t}=\left\{\begin{array}{c}
+1 \text { w.p. } 1 / 2 \\
-1 \text { w.p. } 1 / 2
\end{array}\right.
$$

where $\epsilon_{t}$ are independent random variables as defined above. The probability distribution of $x$ at time $t$ is well known and given by the binomial distribution. Consider now the range of an on-going process and let the range at time $t$ be $R_{t}$,

$$
R_{t}=\operatorname{Max}\left[x_{1}, x_{2}, x_{3}, \cdots, x_{t}\right]-\operatorname{Min}\left[x_{1}, x_{2}, x_{3}, \cdots, x_{t}\right]
$$

A controlled process will have a predictable range process, while an uncontrolled process points out to some unlikely process variation. Practically, range control can help locate and control outliers (which is often very important to do). The range process was first introduced by Feller in 1951 (Feller [2]) who remarked that it is in general difficult to compute the $R_{t}$ distribution for a fixed $t$. Wetherhill [6] indicated that this process has so far not been studied, although Grant and Leavenworth [3] provide some approximation. Since $R_{t}$ is a growing process, we can, equivalently, study its inverse process. Namely, this is the first time that the process has a range which is greater than $n$, or,

$$
\theta(n)=\operatorname{Inf}\left(t \geqq 0 ; R_{t} \geqq n\right)
$$

Clearly, $\left\{R_{n}<a\right\}$ is equal to $\{\theta(a) \geqq n\}$ and therefore the law of $R_{t}$ can be studied equivalently through the probability law of $\theta(a)$. Such a result is given by Vallois [5] who shows that if $X_{\theta(a)}$ denotes the Bernoulli random walk value when the process amplitude (the range) first reaches the value $a$, then the following holds:

$$
P\left(X_{\theta(a)}=k\right)=\frac{|k|}{a(a+1)} ; \quad 0 \leqq|k| \leqq a
$$


Further, if $F(z ; a)$ denotes the probability generating function of the first time the range attains the amplitude $a$, given by

$$
F(z ; a)=\sum_{j=0}^{\infty} P(\theta(a)=j) z^{j}
$$

with,

$$
F(\rho ; a)=E\left[\rho^{\theta(a)}\right]=\frac{2(\alpha+1) \alpha^{a}}{\left(\alpha^{a}+1\right)\left(\alpha^{a+1}+1\right)}
$$

where $\alpha$ is a solution of the following quadratic equation and is therefore a function of $\rho$,

$$
\rho x^{2}-2 x+\rho=0
$$

A proof of these results can be found in Vallois [5]. Our purpose here is to develop an alternative representation of the probability generating function through which we can calculate the expected value and the variance of that first instant when the process attains an amplitude of given size. These two moments can be used to construct approximate tests on the range in a random walk.

Proposition 1: Let $\theta(a)$ be the first instant that a process attains the amplitude $a$, or

$$
\theta(a)=\operatorname{Inf}\left\{t \geqq 0, R_{t} \geqq a\right\}
$$

Then the mean of $\theta(a)$ is given by,

$$
E(\theta(a))=\frac{a(a+1)}{2}
$$

Proof: Since $a$ is fixed we denote by $F(\rho)=F(\rho ; a)$. Let the Probability Generating Function be written in the following form:

$$
\begin{gathered}
F(\rho)=h(\alpha), \quad \alpha=\phi(\rho), \quad \phi(\rho)=\frac{1+\sqrt{1-\rho^{2}}}{\rho} \\
h(\alpha)=\frac{2(\alpha+1) \alpha^{a}}{\left(\alpha^{a}+1\right)\left(\alpha^{a+1}+1\right)}
\end{gathered}
$$

vol. $29, \mathrm{n}^{\circ} 1,1995$ 
Thus, we have

$$
\rho=\frac{2 \alpha}{1+\alpha^{2}}
$$

which implies that

$$
F(\rho)=E\left[\left(\frac{2 \alpha}{1+\alpha^{2}}\right)^{\theta(a)}\right]=h(\alpha)
$$

which we derive to note that

$$
\frac{2\left(1-\alpha^{2}\right)}{\left(1+\alpha^{2}\right)^{2}} E\left[\theta(a)\left(\frac{2 \alpha}{1+\alpha^{2}}\right)^{\theta(a)-1}\right]=h^{\prime}(\alpha)
$$

and since

$$
h^{\prime}(1)=0
$$

The previous equation can be transformed in the following form:

$$
E\left[\theta(a)\left(\frac{2 \alpha}{1+\alpha^{2}}\right)^{\theta(a)-1}\right]=\frac{\left(1+\alpha^{2}\right)^{2}}{2(1+\alpha)}\left(\frac{h^{\prime}(\alpha)-h^{\prime}(1)}{1-\alpha}\right)
$$

and therefore we obtain the equality,

$$
E(\theta(a))=-h^{\prime \prime}(1)
$$

A second derivative of $h(\cdot)$ using L'Hopitale's rule leads at last to $h^{\prime \prime}(1)=-(a(a+1) / 2)$ and to $E(\theta(a))=a(a+1) / 2$ as stated in the proposition.

Q.E.D.

We consider now the second moment. To do so, we calculate another expression of the Probability Generating Function which will eliminate the discontinuity at $\rho=1$. To do so, first we write,

$$
\left[1+\sqrt{1-\rho^{2}}\right]^{n}=a_{n}(\rho)+b_{n}(\rho) \sqrt{1-\rho^{2}}
$$

where

$$
\left.\begin{array}{l}
a_{n}(\rho)=\sum_{2 k \leqq n}\left(\begin{array}{c}
n \\
2 k
\end{array}\right)\left(1-\rho^{2}\right)^{k}, \\
b_{n}(\rho)=\sum_{2 k+1 \leqq n}\left(\begin{array}{c}
n \\
2 k+1
\end{array}\right)\left(1-\rho^{2}\right)^{k}
\end{array}\right\}
$$


Of course, $a_{n}(1)=1$ and $b_{n}(1)=n$. These equations can be written in a recursive form,

$$
\begin{gathered}
a_{n+1}(\rho)=a_{n}(\rho)+b_{n}(\rho)\left(1-\rho^{2}\right) \\
b_{n+1}(\rho)=b_{n}(\rho)+a_{n}(\rho)
\end{gathered}
$$

We shall also use for convenience the added notation,

$$
\begin{gathered}
c_{n}(\rho)=(1+\rho) a_{n}(\rho)+b_{n}(\rho)\left(1-\rho^{2}\right) \\
d_{n}(\rho)=a_{n}(\rho)+(1+\rho) b_{n}(\rho) \\
e_{n}(\rho)=\left(\rho^{n}+a_{n}(\rho)\right)\left(\rho^{n+1}+a_{n+1}(\rho)\right) \\
+\left(1-\rho^{2}\right) b_{n}(\rho) b_{n+1}(\rho) \\
\quad \\
f_{n}(\rho)=b_{n}(\rho)\left(\rho^{n+1}+a_{n+1}(\rho)\right)+b_{n+1}(\rho)\left(\rho^{n}+a_{n}(\rho)\right)
\end{gathered}
$$

Finally, to avoid any confusion, we set $a_{n}=a_{n}(\rho), b_{n}=b_{n}(\rho)$. On the basis of this notation we prove the following proposition.

Proposition 2: The probability Generating Function (7) can be written as follows:

$$
F(\rho ; n)=2 \rho^{n} \frac{d_{n}(\rho)}{f_{n}(\rho)}
$$

and further

$$
e_{n}(\rho) d_{n}(\rho)=c_{n}(\rho) f_{n}(\rho)
$$

Proof: Let $\alpha=\alpha(\rho)$ and thus write, 


$$
\begin{aligned}
(1+\alpha) \alpha^{n} & =\left[\frac{1+\rho+\sqrt{1-\rho^{2}}}{\rho}\right]\left(\frac{a_{n}+b_{n} \sqrt{1-\rho^{2}}}{\rho^{n}}\right) \\
& =\frac{a_{n}(1+\rho)+b_{n}\left(1-\rho^{2}\right)+\left(a_{n}+(1+\rho) b_{n}\right) \sqrt{1-\rho^{2}}}{\rho^{n+1}} \\
& =\frac{c_{n}+d_{n} \sqrt{1-\rho^{2}}}{\rho^{n+1}}
\end{aligned}
$$

Further,

$$
\begin{aligned}
\left(1+\alpha^{n}\right)\left(1+\alpha^{n+1}\right)= & \left(\frac{a_{n}+b_{n} \sqrt{1-\rho^{2}}}{\rho^{n}}+1\right) \\
& \times\left(\frac{a_{n+1}+b_{n+1} \sqrt{1-\rho^{2}}}{\rho^{n+1}}+1\right) \\
= & \frac{1}{\rho^{2 n+1}}\left(\rho^{n}+a_{n}+b_{n} \sqrt{1-\rho^{2}}\right) \\
& \times\left(\rho^{n+1}+a_{n+1}+b_{n+1} \sqrt{1-\rho^{2}}\right)
\end{aligned}
$$

and thus,

$$
\left(1+\alpha^{n}\right)\left(1+\alpha^{n+1}\right)=\frac{1}{\rho^{2 n+1}}\left(e_{n}+f_{n} \sqrt{1-\rho^{2}}\right)
$$

Consequently,

$$
F(\rho ; n)=\frac{2 \rho^{n}\left(c_{n}+d_{n} \sqrt{1-\rho^{2}}\right)}{e_{n}+f_{n} \sqrt{1-\rho^{2}}}
$$

Multiplying through its conjugate value, we obtain,

$$
F(\rho ; n)=\frac{2 \rho^{n}\left(c_{n}+d_{n} \sqrt{1-\rho^{2}}\right)\left(e_{n}-f_{n} \sqrt{1-\rho^{2}}\right)}{e_{n}^{2}-f_{n}^{2}\left(1-\rho^{2}\right)}
$$

or

$$
\begin{aligned}
F(\rho, n)= & 2 \rho^{n}\left[\frac{c_{n} e_{n}-d_{n} f_{n}\left(1-\rho^{2}\right)}{e_{n}^{2}-f_{n}^{2}\left(1-\rho^{2}\right)}\right. \\
& \left.+\sqrt{1-\rho^{2}}\left(\frac{e_{n} d_{n}-c_{n} f_{n}}{e_{n}^{2}-f_{n}^{2}\left(1-\rho^{2}\right)}\right)\right]
\end{aligned}
$$


Now set $Q(\rho)=e_{n}^{2}-f_{n}^{2}\left(1-\rho^{2}\right)$. Since $a_{n}$ and $b_{n}$ are polynomial functions of $\rho, e_{n}$ and $f_{n}$ are necessarily polynomial functions and therefore $Q$ is also a polynomial function with,

$$
Q(1)=e_{n}(1)^{2}=16 \neq 0 .
$$

The rational functions defined above have a non null denominator and therefore the Probability Generating Function in the neighborhood of $\rho=1$ is in class $\mathrm{C}^{\infty}$. As a result, we obtain,

$$
e_{n} d_{n}-c_{n} f_{n}=0
$$

as stated in the proposition. Therefore it is easily shown that

$$
F(\rho ; n)=\frac{2 \rho^{n}\left(c_{n} e_{n}-d_{n} f_{n}\left(1-\rho^{2}\right)\right)}{e_{n}^{2}-f_{n}^{2}\left(1-\rho^{2}\right)}
$$

Since

$$
f_{n}=e_{n} d_{n} / c_{n}
$$

the Probability Generating Function is reduced to the simpler form stated in the Proposition,

$$
F(\rho ; n)=\frac{2 \rho^{n}\left(c_{n} e_{n}-\left(e_{n} d_{n}^{2} / c_{n}\right)\left(1-\rho^{2}\right)\right)}{e_{n}^{2}-\left(e_{n} d_{n}\right)^{2}\left(1-\rho^{2}\right) / c_{n}^{2}}=\frac{2 \rho^{n} c_{n}}{e_{n}}
$$

Since we also have $c_{n} / e_{n}=d_{n} / f_{n}$ the expression stated in the proposition is proved.

Q.E.D.

This new formulation of the Probability Generating Function will now be used to calculate the second moment. Unfortunately, it involves a number of complicated computations to be performed first. For some function $f$, define the following function,

$$
\hat{f}(x)=f(1-x)
$$

Using this notation, we shall compute the functions $\hat{d}_{n}(x), \hat{f}_{n}(x)$ and use,

$$
\hat{F}(x) \hat{f}_{n}(x)=2(1-x)^{n} \hat{d}_{n}(x)
$$

with $\hat{F}(x)=\hat{F}(x ; n)$. Further, based on the polynomial development of these functions we state the following Lemma: 
LemMa 1:

$$
\hat{a}_{n}(x)=\sum_{k=0}^{2 n_{0}} \lambda_{k} x^{k}
$$

where

$$
\begin{gathered}
n_{0}=\left[\frac{n}{2}\right] \\
\lambda_{k}=\sum_{\left(k-n_{0}\right)+\leqq m \leqq k / 2}(-1)^{m} 2^{k-2 m}\left(\begin{array}{c}
n \\
2(k-m)
\end{array}\right)\left(\begin{array}{c}
k-m \\
m
\end{array}\right) \\
\hat{b}_{n}(x)=\sum_{k=0}^{2 n_{1}} \nu_{k} x^{k}
\end{gathered}
$$

where

$$
\begin{gathered}
n_{1}=\left[\frac{n-1}{2}\right] \\
\nu_{k}=\sum_{\left(k-n_{1}\right)+\leqq m \leqq k / 2}(-1)^{m} 2^{k-2 m}\left(\begin{array}{c}
n \\
2(k-m)+1
\end{array}\right)\left(\begin{array}{c}
k-m \\
m
\end{array}\right)
\end{gathered}
$$

Proof: We prove equation (18) first. Since,

$$
\begin{aligned}
\hat{a}_{n}(x) & =\sum_{k=0}^{n_{0}}\left(\begin{array}{c}
n \\
2 k
\end{array}\right)(x(2-x))^{k} \\
& =\sum_{k=0}^{n_{0}}\left(\begin{array}{c}
n \\
2 k
\end{array}\right) x^{k}\left(\sum_{m=0}^{k}\left(\begin{array}{c}
k \\
m
\end{array}\right)(-1)^{m} x^{m} 2^{k-m}\right) \\
& =\sum_{0 \leqq k \leqq n_{0}, 0 \leqq m \leqq k}\left(\begin{array}{c}
n \\
2 k
\end{array}\right)\left(\begin{array}{c}
k \\
m
\end{array}\right)(-1)^{m} x^{k+m} 2^{k-m}
\end{aligned}
$$

By using a change of indices such that $k+m=l$ and $m=m$, we note that $0 \leqq l \leqq 2 n_{0}$. Further, the inequalities $0 \leqq m \leqq n_{0}, m \leqq k=l-m$ and $k=l-m \leqq n_{0}$ is equivalent to the following system $0 \leqq 2 m \leqq l$ and $l-n_{0} \leqq m$ which leads finally to the inequality used in (18), or $\left(l-n_{0}\right)^{+} \leqq m \leqq l / 2$ (where we used $k$ rather than $l$ ). In a similar manner we can proceed and obtain the proof for $\hat{b}_{n}(x)$ as shown in equation (19). 
In particular, note that $\lambda_{0}=1$ and $\nu_{0}=n$. Further, if $n$ is even then $n_{0}=n / 2, n_{1}=\left[\frac{n}{2}-1\right]^{+}$and $n_{0}=n_{1}=(n-1) / 2$ if it is odd.

LEMMA 2 :

$$
\hat{d}_{n}(x)=\sum_{k=0}^{\infty}\left(\lambda_{k}+2 \nu_{k}-\nu_{k-1}\right) x^{k}
$$

with the convention that

$$
\begin{gathered}
\nu_{-1}=0, \quad \lambda_{k}=0, \\
\lambda_{k^{\prime}}=0 \quad \text { for } \quad k>2 n_{0} \quad \text { and } \quad k^{\prime}>2 n_{1}
\end{gathered}
$$

Proof: Since

$$
\hat{d}_{n}(x)=\hat{a}_{n}(x)+(2-x) \hat{b}_{n}(x)
$$

where

$$
\begin{gathered}
\hat{a}_{n}(x)=\sum_{k=0}^{\infty} \lambda_{k} x^{k} \\
\hat{b}_{n}(x)=\sum_{k=0}^{\infty} \nu_{k} x^{k} \\
\hat{d}_{n}(x)=\sum_{k=0}^{\infty}\left(\lambda_{k}+2 \nu_{k}\right) x^{k}-\sum_{k=1}^{\infty} \nu_{k-1} x^{k}
\end{gathered}
$$

as stated in (20).

Q.E.D.

Although it is difficult to compute $\hat{f}_{n}(x)$ for all $n$, we can calculate it for orders up to 2 . To do so, we first note the following results using the above lemma.

LEMMA 3:

$$
\left.\begin{array}{c}
\lambda_{0}=1, \quad \lambda_{1}=n(n-1), \\
\lambda_{2}=\frac{n(n-1)}{6}\left(n^{2}-5 n+3\right), \quad n \geqq 4
\end{array}\right\}
$$




$$
\left.\begin{array}{c}
\nu_{0}=n, \quad \nu_{1}=\frac{n(n-1)(n-2)}{3}, \\
\nu_{2}=\frac{n(n-1)(n-2)}{30}\left(n^{2}-7 n+7\right), \quad n \geqq 5
\end{array}\right\}
$$

Proof: A direct development of (18) leads to (21), while (19) leads to (22) and thus using (20) we obtain (23). Explicitly, we have:

$$
\begin{aligned}
\lambda_{2} & =\sum_{m=0}^{1}(-1)^{m} 2^{2-2 m}\left(\begin{array}{c}
n \\
4-2 m
\end{array}\right) \\
& =4\left(\begin{array}{l}
n \\
4
\end{array}\right)-\left(\begin{array}{l}
n \\
2
\end{array}\right)=\frac{n(n-1)}{6}[(n-2)(n-3)-3]
\end{aligned}
$$

which leads to:

$$
\lambda_{2}=\frac{n(n-1)}{6}\left[n^{2}-5 n+3\right]
$$

as stated in (21).

$$
\begin{aligned}
\nu_{2}= & \sum_{m=0}^{1}(-1)^{m} 2^{2-2 m}\left(\begin{array}{c}
n \\
5-2 m
\end{array}\right) \\
= & 4\left(\begin{array}{l}
n \\
5
\end{array}\right)-\left(\begin{array}{l}
n \\
3
\end{array}\right)=\frac{n(n-1)(n-2)}{5 \cdot 3 !} \\
& \times[(n-3)(n-4)-5] \\
= & \frac{n(n-1)(n-2)}{30}\left(n^{2}-7 n+7\right), \quad n \geqq 5 .
\end{aligned}
$$

Finally, to compute (23), note that

$$
\begin{aligned}
\lambda_{1}+2 \nu_{1}-\nu_{0} & =n(n-1)+\frac{2 n(n-1)(n-2)}{3}-n \\
& =\frac{n}{3}(n-2)(2 n+1)
\end{aligned}
$$

and 


$$
\begin{aligned}
\lambda_{2}+2 \nu_{2}-\nu_{1}= & \frac{n(n-1)}{6}\left(n^{2}-5 n+3\right) \\
& +\frac{n(n-1)(n-2)}{15}\left(n^{2}-7 n+7\right) \\
& -\frac{n(n-1)(n-2)}{3}=\frac{n(n-1)}{30} \\
& \times\left(2 n^{3}-13 n^{2}+7 n+7\right)
\end{aligned}
$$

Q.E.D.

LEMMA 4:

$$
\begin{aligned}
\hat{f}_{n}(x)= & 2(2 n+1)+\frac{n}{3}\left(10 n^{2}-9 n-7\right) x \\
& +\frac{n(n-1)}{30}\left(34 n^{3}-81 n^{2}-n+24\right) x^{2}+0\left(x^{2}\right)
\end{aligned}
$$

Proof: As stated earlier [equation (13f)], we have

$$
\hat{f}_{n}(x)=\hat{b}_{n}(x)\left(\hat{a}_{n+1}(x)+(1-x)^{n+1}\right)+\hat{b}_{n+1}(x)\left(\hat{a}_{n}(x)+(1-x)^{n}\right)
$$

where

$$
\begin{aligned}
& \hat{a}_{n}(x)=1+n(n-1) x+\frac{n(n-1)}{6}\left(n^{2}-5 n+3\right) x^{2}+0\left(x^{2}\right) \\
& \hat{a}_{n+1}(x)+(1-x)^{n+1}=2+\left(n^{2}-1\right) x+\frac{(n+1) n(n-1)(n-2)}{6} x^{2}+0\left(x^{2}\right) \\
& \hat{b}_{n}(x)=n+\frac{n(n-1)(n-2)}{3} x+\frac{n(n-1)(n-2)}{30}\left(n^{2}-7 n+7\right) x^{2}+0\left(x^{2}\right) \\
& \hat{b}_{n}(x)\left(\hat{a}_{n+1}(x)+(1-x)^{n+1}\right) \\
& \quad=n\left[2+\frac{(n-1)(5 n-1)}{3} x+\frac{(n-1)(n-2)}{30}\left(17 n^{2}-9 n+4\right) x^{2}\right] \\
& \quad+0\left(x^{2}\right)
\end{aligned}
$$




$$
\begin{aligned}
& \hat{b}_{n+1}(x)\left(\hat{a}_{n}(x)+(1-x)^{n}\right) \\
&=(n+1)\left[2+\frac{n}{3}(5 n-8) x\right. \\
&\left.\quad+\frac{n(n-1)}{30}\left(17 n^{2}-55 n+32\right) x^{2}\right]+0\left(x^{2}\right)
\end{aligned}
$$

Inserting these equations above in $\hat{f}_{n}(x)$, we obtain equation (24).

Q.E.D.

Finally, we turn to Lemma 5 which will be used subsequently to compute the variance.

LEMMA 5:

$$
E(\theta(n)(\theta(n)-1))=\frac{(n-1) n(n+1)(n+2)}{3}
$$

Proof: We begin by letting $\hat{F}_{n}(x ; n)=1+\gamma_{1} x+\gamma_{2} x^{2}+O\left(x^{2}\right)$. From equation (17) we derive,

$$
\begin{gathered}
\hat{F}(x ; n) \hat{f}_{n}(x)=2(2 n+1)+\left\{2 \gamma_{1}(2 n+1)\right. \\
\left.+\frac{n}{3}\left(10 n^{2}-9 n-7\right)\right\} x+\tau_{1} x^{2}+O\left(x^{2}\right) \\
\left.\tau_{1}=2 \gamma_{2}(2 n+1)+\frac{n}{3}\left(10 n^{2}-9 n-7\right)\right\} \gamma_{1} \\
\quad+\frac{n(n-1)}{30}\left(34 n^{3}-81 n^{2}-n+24\right) \\
{[1-x]^{n}=1-n x+\frac{n(n-1)}{2} x^{2}+O\left(x^{2}\right)} \\
\tau_{2}=\frac{n(n-1)}{30}\left(2 n^{3}-13 n^{2}+7 n+7\right) \\
-\frac{n^{2}}{3}(n-2)(2 n+1)+\frac{n(n-1)}{2}(1+2 n)
\end{gathered}
$$


This leads to the following equations,

$2 \gamma_{1}(2 n+1)+\frac{n}{3}\left(10 n^{2}-9 n-7\right)=2\left(\frac{n}{3}(n-2)(2 n+1)-n(1+2 n)\right)$

and

$$
\tau_{1}=2 \tau_{2}
$$

The first equation leads to,

$$
2 \gamma_{1}(2 n+1)=\frac{n}{3}\left(-10 n^{2}+9 n+7+4 n^{2}-6 n-4-6-12 n\right)
$$

and after its solution to:

$$
\gamma_{1}=-\frac{n(n+1)}{2}
$$

As noted earlier, $F^{\prime}(1 ; n)=-\hat{F}^{\prime}(0 ; n)=-\gamma_{1}$, providing thereby an expression for the mean. For the second equation, this leads to, $2(2 n+1) \gamma_{2}=(n / 30) \tau_{3}$, where

$$
\begin{aligned}
\tau_{3}= & 2(n-1)\left(2 n^{3}-13 n^{2}+7 n+7\right) \\
& -20 n(n-2)(2 n+1)+30(n-1)(2 n+1) \\
& +5\left(10 n^{2}-9 n-7\right) n(n+1)-(n-1)\left(34 n^{3}-81 n^{2}-n+24\right)
\end{aligned}
$$

Since $10 n^{2}-9 n-7=(2 n+1)(5 n-7)$, extensive manipulations of the previous identity lead to:

$$
\gamma_{2}=\frac{n}{30} \tau_{3} \frac{1}{2(2 n+1)}=\frac{1}{6} n\left(n^{2}-1\right)(n+2)
$$

and finally to:

$$
F^{\prime \prime}(1 ; n)=\hat{F}^{\prime \prime}(0 ; n)=2 \gamma_{2}=E(\theta(n)(\theta(n)-1))
$$

Q.E.D.

With these Lemma, Proposition 3 provides the variance estimate of $\theta(a)$. 
Proposition 3: Let $\theta(a)$ be again the first instant that a process attains the amplitude a, then its variance is given by,

$$
\operatorname{Var}(\theta(a))=\frac{(a-1) a(a+1)(a+2)}{12}
$$

Proof: Using the previous results, the proof of this proposition is straightforward. Note that

$$
\begin{aligned}
E\left(\theta(a)^{2}\right)= & E(\theta(a)(\theta(a)-1))+E(\theta(a))=\frac{a}{3}\left(a^{2}-1\right)(a+2) \\
& +\frac{a(a+1)}{2}=\frac{a(a+1)}{6}\left[2 a^{2}+2 a-1\right]
\end{aligned}
$$

As a result, the variance of $\theta(a)$ is given by,

$$
\begin{aligned}
\operatorname{Var}(\theta(a)) & =\frac{a(a+1)}{6}\left[2 a^{2}+2 a-1\right]-\left[\frac{a(a+1)}{2}\right]^{2} \\
& =\frac{(a-1) a(a+1)(a+2)}{12}
\end{aligned}
$$

as stated in the proposition.

Q.E.D.

Using these two propositions it is possible to standardize the distribution. Explicitly, consider the following random variable which is divided by its mean. The mean will therefore equal one, while the variance is

$$
\frac{\operatorname{Var}(\theta(a))}{[E(\theta(a))]^{2}}=\frac{(a-1)(a+2)}{3 a(a+1)}
$$

When a increases, this variance tends to $1 / 3$. For example, for an amplitude of 9 , this variance equals already (1/3) (88/90). This standardization can of course be used to construct Tables and use approximations using other distributions. If the unit for the amplitude is very small, we obtain approximately,

$$
\operatorname{Var}(\theta(a))=\frac{a^{4}}{12}, \quad E(\theta(a))=\frac{a^{2}}{2} \quad \text { and } \quad \frac{\operatorname{Var}(\theta(a))}{[\mathrm{E}(\theta(\mathrm{a}))]^{2}}=\frac{1}{3}
$$

In order to test the validity of these results extensive simulations were performed, the mean and the variances calculated. The results obtained have confirmed the theoretical results derived here. Of course, using simulation, higher order moments can be computed and the simulated probability 
distributions constructed. These can be used to construct statistical tables as well as test approximations using simpler and well documented distributions (such as Weibull, Gama, inverse Gaussian distributions and distributions with three or more parameters).

\section{APPLICATIONS}

\section{Exchange Rate Controls}

Let $x_{t}$ be an exchange rate process and assume that we seek to control the process variability through measurement of the time consumed to reach a given amplitude. In other words, let there be some exchange rate (amplitude) variation $R$ observed at some given time $t$. Can we infer that this observation is unexpected? Similarly, given bounds for exchange rate variations, we can estimate the "normal" time for the exchange rate to exit these bounds (and thus require the intervention of the Central Bank). Say that measurements occur in the time interval $(0, t)$ and let the maximal amplitude be $A$ observed at time $T$. Standardize this observation by dividing by $A(A+1) / 2$. Namely, we record $2 T / A(A+1)$. For discussion purposes, assume that we use a Weibull approximation with parameters $\alpha$ and $c$ (appropriately computed by equating the first two moments),

$$
E(2 t / A(A+1))=\alpha \Gamma(1 / c+1)=1
$$

and

$$
E(2 t / A(A+1))^{2}=\alpha^{2} \Gamma(2 / c+1)=1+\frac{(A-1)(A+2)}{3 A(A+1)}
$$

Then the probability of recording an amplitude $A$ before or a time $T$ is given by:

$$
P(x \leqq y)=\exp \left[-\left(\frac{y}{\alpha}\right)^{c}\right], \quad \text { where } \quad y=2 T / A(A+1)
$$

which can be used to construct confidence intervals and test hypotheses regarding the occurrence of amplitudes in exchange rate variations. Further research is required however, using appropriate stochastic models of exchange rates variations. 


\section{Statistical Tolerance Limits}

Statistical tolerance limits are defined by:

$$
\operatorname{Prob}\left[\int_{a}^{b} f(x ; \theta) d x \geqq \delta\right]=\alpha
$$

with $a$ and $b$ random, are an important tool of quality control. These limits are used to indicate with a given probability $\alpha$ the amount of variability that one can expect in at least $100 \delta$ percent of the specimen tested in a production process. As a result, the statistical tolerance limits provide an estimate of the process variability. Of course, to calculate the distribution of $(a, b)$, the amplitude $(b-a)$ distribution is required. Then,

$$
E_{a, b} \operatorname{Prob}\left[\int_{\mathrm{a}}^{b} f(x ; \theta) d x \geqq \delta\right]=\alpha
$$

When the process is described by a random walk, this approach is no longer feasible. Using our results, we can define a statistical tolerance limit as the probability for the amount of time needed to attain a given boundary. The larger this time, the more stable the process and vice versa (assuming of course, that the process is controlled in the mean). Explicitly, for an amplitude $y$, the probability that this amplitude is reached prior to $T$ is, $P(\theta(y) \leqq T)$. Thus if $Y$ is a maximal (and tolerable) amplitude, the probability that the amplitude will be smaller than $Y$ in a given time interval $T$ is given by

$$
\int_{0}^{Y} P(\theta(y) \leqq T) d y
$$

which provides an alternative measure of process stability. These and other applications such as the detection of outliers, inventory control and so on provide important motivation for the study of amplitude processes in random walks and will be considered in subsequent research.

\section{ACKNOWLEDGEMENTS}

The authors are grateful to Jean-Jacques Daudin for useful comments as well as support from the CERESSEC and EEC Contract ERB, 4050 PL930200 on Decision Support for Maintenance Management and Quality Control (Human Capital and Mobility Program, 1993-1995). 


\section{REFERENCES}

1. C. S. van DobBen de Bruyn, Cumulative Sum Tests: Theory and Practice, Griffin, London, 1968.

2. W. Feller, The asymptotic distribution of the range of sums of independent random variables, Annals of Math. Stat., 1951, 22, pp. 427-432

3. E. L. Grant and R. S. Leavenworth, Statistical Quality Control, 6th ed., McGraw Hill, 1988.

4. C. S. TAPIERo, The Management of Quality and Its Control, Chapman and Hall, London, 1994, Forthcoming.

5. P. Vallois, On the range process of a Bernoulli random walk, Proceedings of the Sixth International Symposium on Applied Stochastic Models and Data Analysis, vol. I, Editors, J. JANSSEN and C. H. SkiAdAs, World Scientific, pp. 1020-1031.

6. G. B. WetherhiLl, Sampling Inspection and Quality Control, Chapman and Hall, London, 1977. 